\title{
Dermopatía fibrosante nefrogénica: Reporte de dos casos
}

\author{
Marcela Concha $\mathbf{R}^{1}$, Cristián Vera $\mathrm{K}^{\mathbf{1}}$, Aquiles Jara $\mathrm{C}^{2}$, \\ Fernando Atabales $A^{a}$, Sergio González $B^{3}$. \\ Nephrogenic fibrosing dermopathy. \\ Report of two cases
}

\begin{abstract}
Nephrogenic fibrosing dermopathy is a recent designation to describe cutaneous findings in patients with renal disease who developed scleromyxedema-like skin lesions with thickening and hardening of the skin. These skin lesions appear mainly after hemodialysis or renal transplantation but their origin is still unknown. We report a 63 years old male on hemodialysis and 56 years old male on peritoneal dialysis, who developed a nephrogenic fibrosing dermopathy that was confirmed by pathology and immunohistochemistry with monoclonal antibodies against CD34 (Rev Méd Chile 2007; 135: 640-6).

(Key words: Hemodyalisis; Nephrogenic fibrosing dermopathy; Peritoneal dyalisis; Scleromyxedema)

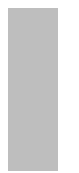

Recibido el 20 de julio, 2006. Aceptado el 22 de agosto, 2006.

${ }^{1}$ Departamento de Dermatología; ${ }^{2}$ Departamento de Nefrología; ${ }^{3}$ Departamento de Anatomía Patológica, Facultad de Medicina, Pontificia Universidad Católica de Chile. Santiago de Chile.

anterno
\end{abstract}

$\mathrm{L}$ a dermatopatía fibrosante nefrogénica (DFN) es la designación recientemente incorporada, que se utiliza para describir los hallazgos cutáneos en pacientes con insuficiencia renal que presentan lesiones similares a escleromixedema con engrosamiento e induración de la piel no asociadas a paraproteinemia.

Reportada por primera vez en 1997 por Cowper et al, quienes en un comienzo la denominaron "Enfermedad escleromixedematosa-símil de la hemodiálisis $"{ }^{1}$. Describieron 14 pacientes con insuficiencia renal de diferentes etiologías, con engrosamiento e induración cutánea de las extremidades, asociado a hiper-

Correspondencia a: Dra. Marcela Concha R. Vicuña Mackenna 4687. Macul, Santiago, Chile. Fono 56-23548492. E mail: marcelaconcha@gmail.com pigmentación y, en algunos casos pápulas y nódulos subcutáneos sin compromiso facial. Todos estos pacientes habían recibido o estaban recibiendo hemodiálisis y 9 de los 14 pacientes llegaron al trasplante renal. En esta primera serie de pacientes, los rangos de edad variaban desde los 31 a los 74 años, siendo más frecuente en el sexo masculino y presentándose en todas las razas.

Los pacientes desarrollan la enfermedad en forma progresiva, en pocos días a semanas. Las lesiones típicas se describen como edema, eritema, seguidas de placas eritematosas de color café, pápulas y nódulos en las extremidades. En forma excepcional se describen bulas y contractura de las articulaciones. Una de las características clínicas relevantes son prurito y dolor urente, asociado a veces a un aumento de la temperatura local ${ }^{2}$.

Actualmente se han descrito más de 170 casos de esta patología en Norteamérica, Europa y 
$\mathrm{Asia}^{3-5}$. En contraste con la descripción original de un síndrome netamente cutáneo, informes recientes indican que puede existir compromiso sistémi$\mathrm{co}^{6-8}$.

Presentamos dos casos de DFN en pacientes con insuficiencia renal en hemodiálisis actual y que representan los primeros casos comunicados en Chile.

Caso 1. Paciente de sexo masculino de 63 años con antecedentes de nefrectomía derecha por hipernefroma, hipertensión arterial, un episodio de trombosis venosa profunda secundario a ruptura del tendón de Aquiles. El paciente estaba en hemodiálisis desde 1 año antes a causa de una insuficiencia renal secundaria a un episodio de rabdomiólisis.

$\mathrm{Al}$ examen físico presentaba una placa eritemato-violácea de $12 \mathrm{~cm}$ de diámetro en flanco izquierdo, asintomática, asociada a aumento de temperatura y eritema de ambas piernas. Dado el antecedente de inicio de gabapentina por un síndrome de Sudeck (distrofia simpática refleja), se sospechó como probables una reacción medicamentosa fija o una celulitis, solicitándose una biopsia cutánea.

Caso 2. Hombre de 56 años con antecedentes de insuficiencia renal crónica secundaria a hipertensión arterial, en hemodiálisis por 23 años, nefrectomía bilateral por carcinoma de células renales 1 año antes y, al consultar, en diálisis peritoneal. El paciente consultó por placas induradas palmoplantares sensibles al tacto, de un año de evolución, que se erosionaron y presentaban dolor urente e impotencia funcional secundaria, con imposibilidad de empuñar y flectar las manos y rigidez de ambas rodillas y codos.

Al examen físico destacaban cambios esclerodermiformes en manos, palmas y plantas asociadas a infartos en pulpejos. Se realizó biopsia cutánea con sospecha de dermatopatía fibrosante nefrogénica.

Ambos estudios histológicos fueron compatibles con dermatopatía fibrosante nefrogénica con acentuada fibrosis de la dermis, superficial y profunda, constituida por bandas gruesas de colágeno, desordenadas, con depósitos de mucina intersticial, leve infiltrado linfocitario perivascular, superficial y profundo, y numerosas células fusa- das intersticiales, difusamente distribuidas. No se encontró vasculitis ni granulomas. El estudio inmunohistoquímico con anticuerpos monoclonales para CD34 mostró reacción positiva en las células fusadas entre los haces de colágeno. Se realizó también tinción de fibras elásticas mostrando reacción positiva.

\section{DisCUSIÓN}

Los casos descritos representan los primeros casos de dermopatía fibrosante nefrogénica descritos en nuestro país. La forma de presentación clínica es característica y los hallazgos histopatológicos son diagnósticos.

La DFN descrita inicialmente por Cowper y colaboradores, se presenta en pacientes con cierto grado de insuficiencia renal y mayoritariamente en pacientes con enfermedad renal terminal en hemo o peritoneodiálisis ${ }^{1}$. Se caracteriza por la aparición de edema y eritema de la piel que progresa a engrosamiento e induración, formando placas eritematosas o de color café, asociadas, a veces, a pápulas, nódulos o bulas. Las lesiones son por lo general bilaterales y simétricas, presentándose predominantemente en extremidades, ya sea entre las rodillas y la mitad del muslo, o entre las muñecas y la mitad de los brazos. En forma ocasional compromete las manos, los pies y el tronco?. A diferencia de lo que ocurre con el escleromixedema, éste respeta la cara y el cue$1 \mathrm{o}^{10}$.

Las placas son firmes, con un borde irregular distintivo y con proyecciones ameboideas digitiformes y con islas de piel sana (Figura 1) ${ }^{10}$. El engrosamiento y la tensión secundaria pueden provocar rigidez, comprometiendo la flexión y extensión de las articulaciones (Figura 2) con la consecuente invalidez a la deambulación.

Los principales síntomas son prurito y dolor urente, mientras que la fiebre, las mialgias o la artritis no se presentan con regularidad ${ }^{11}$.

El diagnóstico se sospecha en forma clínica, pero su confirmación es mediante el estudio histopatológico. En la biopsia se observa un engrosamiento de las bandas colágenas en la dermis reticular (Figura 3), aumento de las fibras elásticas, presencia de abundantes fibroblastos en forma difusa, fibrosis subcutánea y de la dermis, 


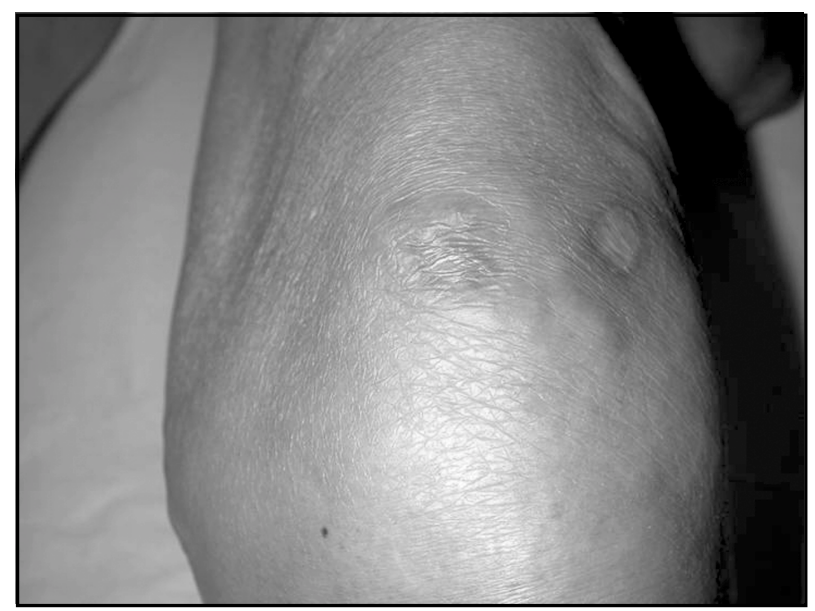

Figura 1. Placa de borde irregular y proyecciones ameboideas digitiformes.

Figura 2. Imposibilidad de flexo-extensión de las articulaciones.

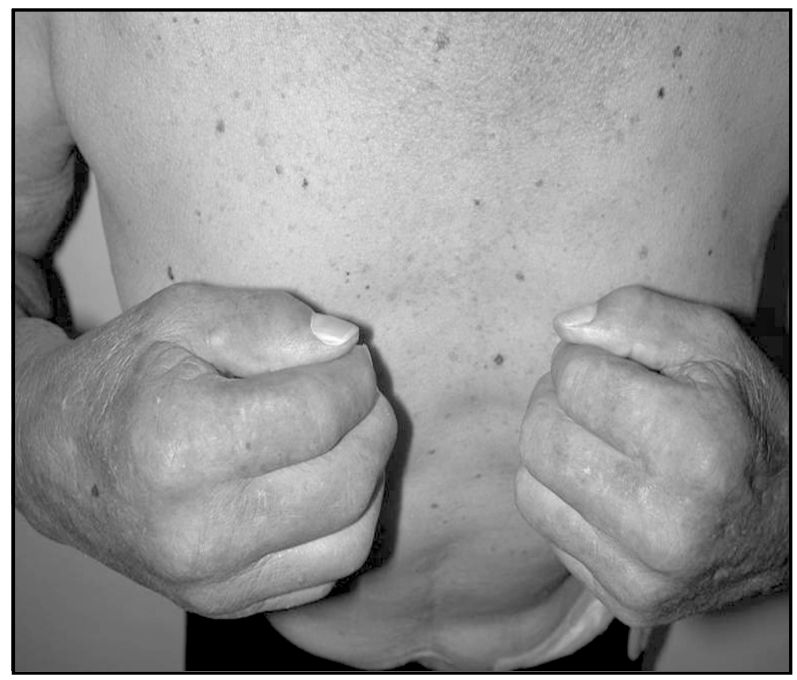

engrosamiento de tabiques y fascias subcutáneas con extensión a músculos esqueléticos, depósitos de mucina en la dermis y depósitos de calcio entre las bandas de colágeno, sin calcificación de los vasos $^{12}$. Otros hallazgos son la atrofia de miofibras, y la presencia de células estrelladas en la dermis, fascias y músculo con inmunohistoquímica positiva para CD34 y procolágeno I, así como para el factor XIIIa, y CD68 positivo en células mono y multinucleadas $2,3,11$.

La descripción original de Cowper et al, describía sólo lesiones locales, en la actualidad existen reportes de DFN con compromiso sistémi- $\operatorname{co}^{6,8,12}$, que describen fibrosis extensa y calcificación de diafragma, psoas, túbulos renales y rete testis.

Si bien no existen hallazgos de laboratorios característicos, la mayoría de las series de casos coinciden con la ausencia de eosinofilia periférica y de paraproteinemia pesquisable a la electroforesis de proteínas ${ }^{4,10}$. Se describen pruebas tiroideas normales, anticuerpos antinucleares positivos pero sin síntomas ni signos de lupus eritematoso sistémico. Recientemente, existen algunos reportes con presencia de anticuerpos anticardiolipinas $\mathrm{o}$ antifosfolípidos positivos ${ }^{10}$. 


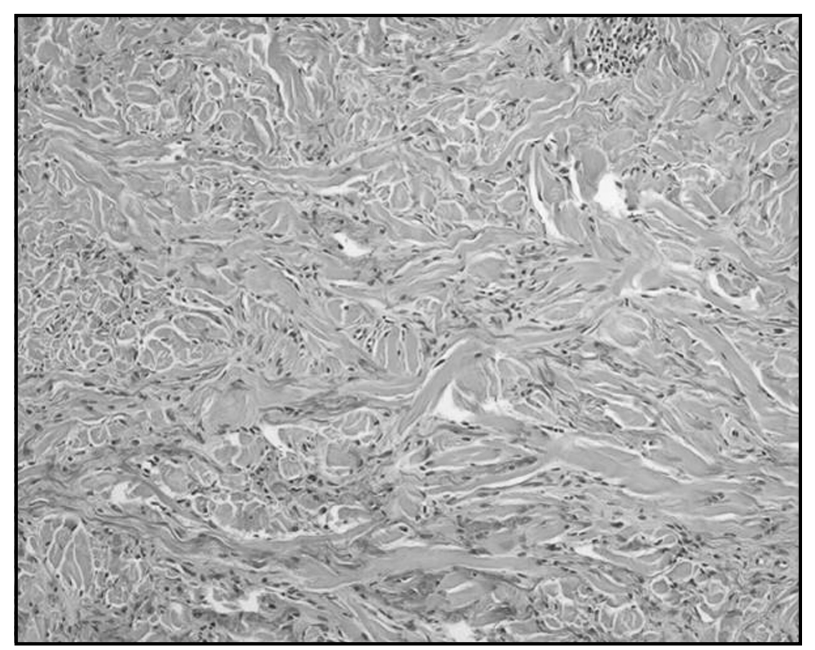

Figura 3. Engrosamiento de las bandas colágenas en la dermis reticular aumento de las fibras elásticas, presencia de abundantes fibroblastos en forma difusa.

Epidemiología actual. No existe predisposición racial, reportándose casos en pacientes asiáticos, afroamericanos, hispanos y anglosajones. Si bien los primeros reportes por Cowper informaban mayor prevalencia de la enfermedad en pacientes de sexo masculino, actualmente registros internacionales notifican la enfermedad en ambos sexos en una razón de 1:1. La enfermedad ha sido descrita en pacientes adultos y pediátricos, con un rango de edad de 8-87 años y una edad promedio de 46,4 años ${ }^{13,14}$.

Diagnóstico diferencial. El diagnóstico diferencial más frecuente es el escleromixedema ${ }^{10}$, una variante del liquen mixedematoso, caracterizada por la presencia de placas y pápulas induradas que clínicamente son similares a la DFN, pero que a diferencia de ésta, compromete la cara y área cervical, siendo el compromiso de manos y pies poco frecuente. Los bordes de las placas no presentan bordes tan netos, ni proyecciones ameboideas. Presentan además paraproteinemia positiva a cadenas livianas. El compromiso sistémico es común, comprometiendo el sistema cardíaco, gastrointestinal y neurológico. A la histología la diferencia principal con la DFN es la presencia de células inflamatorias y plasmáticas ${ }^{15}$.

La escleroderma se presenta con esclerosis, tensión e induración de la piel. El fenómeno de
Raynaud y el compromiso sistémico se manifiestan en forma habitual, a diferencia de la DFN. Típicamente presenta anticuerpos antinucleares, anticentrómeros y anti-Scl-70 positivos ${ }^{15}$. A la histología destaca una disminución de las células a nivel de la dermis, e inflamación perivascular. La morfea, una variante localizada de la escleroder$\mathrm{ma}$, se presenta con placas induradas en el tronco y no se asocia a síntomas sistémicos. A la histología destaca una infiltración linfocitaria intersticial y perivascular.

La fasceítis eosinofílica se presenta con dolor, edema, eritema e induración de las extremidades después de un episodio de esfuerzo moderado. El compromiso de tronco o sistémico es habitual. Destacan las contracturas musculares y el compromiso de las fascias. La esclerodermia es un estadio final de la enfermedad. Al laboratorio destaca la eosinofilia periférica, el aumento de la VHS, y la hipergamaglobulinemia. Histológicamente se caracteriza por presentar inflamación de la fascia, edema, esclerosis e infiltrado inflamatorio con linfocitos, histiocitos, células plasmáticas, y eosinófilos. A diferencia de la DFN, en la fascé́tis eosinofílica el depósito de mucina no es tan marcado, y el infiltrado inflamatorio es polimorfo ${ }^{15,16}$.

El síndrome eosinofilia mialgia se describió en Estados Unidos de Norteamérica en 1989, en 
pacientes que tomaron triptófano contaminado para el insomnio ${ }^{17,18}$. Se presenta en forma aguda con mialgia intensa, eosinofilia, urticaria, disnea, y síntomas sistémicos. La mitad de los pacientes desarrollan lesiones esclerodermiformes. La biopsia cutánea se caracteriza por presentar bandas de colágeno gruesas, y a diferencia de la DFN, el depósito de mucina no ocurre entre las bandas de colágenos, sino más bien en lagunas y el infiltrado inflamatorio es perivascular y compuesto por linfocitos, células plasmáticas, macrófagos y eosinófilos.

La ingestión tóxica de aceite o el síndrome tóxico de aceite, fue una epidemia que ocurrió en España en 1981 por la ingestión de aceites adulterados, casos nuevos no existen reporta$\operatorname{dos}^{16}$.

\section{ETIOLOGÍA}

La causa de la DFN es desconocida y parece ser multifactorial. El hecho que la enfermedad se haya presentado en pacientes no sometidos a diálisis o a trasplante renal, sugiere que la DFN es más bien una patología asociada a insuficiencia renal sin importar la causa desencadenante de ésta ${ }^{10}$, sin embargo, el aumento de casos en los últimos años sugiere que podrían existir factores exógenos asociados (cambios recientes de medicación, técnicas quirúrgicas o de equipos).

Estudios inmunohistoquímicos revelan que el componente principal de las lesiones de la DFN, son células fusadas CD34 y procolágeno positivas, que corresponden inmunofenotípicamente a fibrocitos circulantes que se reubicarían en la dermis ${ }^{11}$. De acuerdo a esta teoría, la DFN correspondería a una enfermedad con reclutamiento, activación y proliferación aberrante de fibrocitos ${ }^{19}$. Por esta razón, en la actualidad, algunos autores proponen el uso de fibrosis nefrogénica sistémica para referirse a la $\mathrm{DFN}^{20}$.

Los inhibidores de la calcineurina han sido implicados en la iniciación de la fibrosis de los tejidos, dado su capacidad de inducir altos niveles de una citoquina profibrótica, el TGFß ${ }^{1}$ (transforming growth factor $\left.\aleph^{1}\right)^{21,22}$.

Algunos autores relacionan la falta de uso de inhibidores de enzima de conversión de la angiotensina (IECA) con la aparición de la enferme- $\mathrm{dad}^{22}$. Estas observaciones se basan en el hecho de que los IECA han demostrado tener un efecto protector en contra de la fibrosis en distintos modelos y a que muchos de los pacientes que presentaban DFN no utilizaban IECA, a pesar de que la mayoría de los pacientes en insuficiencia renal lo hacía. Se cree que el efecto de la angiotensina II estaría mediado por su transformación en el TGFß ${ }^{1}$, interleukina 1 y otros mediadores, favoreciendo así la proliferación fibroblástica. Reportes recientes avalan esta hipótesis, correlacionando los bajos niveles del TGF $\beta^{1}$ después de la plasmaféresis con la mejoría clínica en 2 pacientes $^{23}$, sin embargo, aún existe controversia al respecto.

Estudios epidemiológicos en algunas series encontraron a la cirugía vascular, la trombosis y los tumores vasculares como eventos precursores de esta enfermedad ${ }^{11}$. Un factor común denominador de todas estas comorbilidades, es el uso de agentes para angiografía, lo que ha permitido que algunos grupos de estudios investiguen a los medios de contraste radiográficos como factores gatillantes de la DFN. Se cree que el depósito periférico de medios de contrastes podrían ser un blanco para los fibrocitos circulantes ${ }^{24}$. Incluso, grupos austríacos han detectado que el gadoliniuo, un medio de contraste para la angiorresonancia, ha sido utilizado en todos los pacientes con DFN de su grupo de estudio. Sin embargo, vieron que pacientes en insuficiencia renal que no presentaron DFN y que sí fueron expuestos al gadoliniuo, tenían en común no haber estado acidóticos en el momento de la exposición, lo que ha llevado a suponer la necesidad de un estado basal acidótico, más la exposición al medio de contraste para el desarrollo de la enfermedad ${ }^{25}$.

El curso de esta enfermedad es incierto y no se correlaciona con la evolución de la enfermedad renal ni con la duración de la insuficiencia renal, ocurriendo tanto al principio como años después de diagnosticada la insuficiencia renal. Se ha descrito el antecedente de anasarca o edema cutáneo previo al inicio de la aparición de lesiones ${ }^{10}$.

\section{TRATAMiento}

Las diferentes publicaciones indican que no existe una terapia efectiva probada, y que el pronóstico 
depende de la extensión, severidad y rapidez del compromiso cutáneo y sistémico ${ }^{4}$. Hasta $28 \%$ de los enfermos mueren, $28 \%$ no mejoran y $20 \%$ tienen una mejoría parcial. Sólo un porcentaje escaso de los pacientes mejoran luego de que la función renal mejora y cesa la diálisis $2,11,12,27-31$.

La terapia física juega un rol preponderante en la recuperación de la movilidad y en la prevención de morbilidad a largo plazo.

La plasmaféresis ha sido el tratamiento más utilizado, sin embargo, sus resultados son controversiales. Es importante hacer notar que aquellos pacientes que tuvieron una mejoría moderada $\mathrm{o}$ importante, son pacientes que discontinuaron la diálisis, dado que su función renal había mejora$\mathrm{do}^{10,21,23}$. En algunos pacientes, el uso de interferón o de inmunoglobulinas endovenosas ha mostrado ser parcialmente efectivo, sin embargo, en la mayoría de los casos los efectos son sólo momentáneos $^{21,32}$. Los resultados con la fotoféresis extracorpórea son controversiales $6,27,30,32$, al igual que otros tratamientos como corticoesteroides (tópicos o sistémicos), ciclosporina, metotrexato ${ }^{6,21,29,31}$, calcipotrienos, ciclofosfamida, talidomida, tacroli-

\section{REFERENCIAS}

1. Cowper Se, Robin HS, Steinberg SM, Su LD, Gupta $S$, LeBort PE. Scleromixedema-like cutaneous diseases in renal-dialysis patients. Lancet 2000; 356: 1000-1.

2. Cowper SE, Su LD, Bhawan J, Robin HS, LeBoit PE. Nephrogenic Fibrosing Dermopathy. Am J Dermatopathol 2001; 23: 383-93.

3. COWPer SE, Boyer PJ. Nephrogenic systemic fibrosis: an update. Curr Rheumatol Rep 2006; 8: 151-7.

4. Mendoza FA, Artlett CM, Sandorfi N, Latinis K, Piera-Velázquez S, Jiménez SA. Description of 12 cases of nephrogenic fibrosing dermopathy and review of the literature. Semin Arthritis Rheum 2006; 35: 238-49.

5. Panda $\mathrm{S}$, Bandyopadhyay D, Tarafder A. Nephrogenic fibrosing dermopathy: a series in a nonWestern population. J Am Acad Dermatol 2006; 54: 155-9.

6. Jiménez SA, Arlett CM, Sandorfi N, Derk C, Latinis K, SaWaYa H et al. Dialysis-Associated Systemic mus, micofenolato, clorambucil, retinoides orales, psoralenos y radiación ultravioleta.

\section{PRONÓstico}

La dermatopatía fibrosante nefrogénica es un diagnóstico poco frecuente, cuya historia natural se desconoce. Algunos pacientes obtienen una mejoría gradual de su movilidad cutánea y articular, con el tiempo. No se ha reportado una mejoría total de la enfermedad en pacientes que persisten en insuficiencia renal.

Muchos de los pacientes con DFN han muerto por las complicaciones de su insuficiencia renal o por la cirugía de trasplante. Un paciente que discontinuó la diálisis tuvo un compromiso sistémico de la enfermedad con fibrosis del diafragma secundaria ${ }^{8}$.

Aun cuando la DFN no ha sido señalada como una causa de muerte, la inmovilidad provocada por esta enfermedad, lleva a caídas y fracturas que llevan a un aumento de morbilidad y mortalidad secundaria.
Fibrosis (Nephrogenic Fibrosing Dermopathy). Arthritis Rheum 2004; 50: 2660-66.

7. Levine JM, Taylor RA, Elman LB, Bird SJ, Lavi E, STOlzENBERG ED. Involvement of skeletal muscle in dialysis-associated fibrosis (Nephrogenic Fibrosing Dermopathy). Muscle \& Nerve 2004; 30: 56977.

8. Kucher C, Steere J, Elenitsas R, Siegel DL, Xu X. Nephrogenic fibrosing dermopathy/nephrogenic systemic fibrosis with diaphragmatic involvement in a patient with respiratory failure. J Am Acad Dermatol 2006; 54: S31-4.

9. Markus JS, James AJ, Nunez-Gussman JK, Sheehan AM, Fegan L, Hsu S. Nephrogenic fibrosing dermopathy. J Am Acad Dermatol 2005; 52: 166-7.

10. Mackay-Wiggan JM, Cohen DJ, Hardy MA, Knobler EH, Grossman ME. Nephrogenic fibrosing dermopathy (scleromyxedema-like illness of renal disease). J Am Acad Dermatol 2003; 48: 55-60.

11. COWPER SE. Nephrogenic fibrosing dermopathy: the first 6 years. Curr Opin Rheumatol 2003; 15 : 785-90. 
12. Ting WW, Stone MS, Madison KC, Kurtz K. Nephrogenic fibrosing dermopathy with systemic involvement. Arch Dermatol 2003; 139: 903-6.

13. Jain SM, Wesson S, Hassanein A, Canova E, Hoy M, FenNell RS ET AL. Nephrogenic fibrosing dermopathy in pediatric patients. Pediatr Nephrol 2004; 19: 467-70.

14. Di Carlo JB, Gupta EA, Solomon AR. A pediatric case of nephrogenic fibrosing dermopathy: improvement after combination therapy. J am Acad Dermatol 2006; 54: 914-6.

15. Perazella ma, Ishibe $S$, Perazella ma, Reilly RF. Nephrogenic fibrosing dermopathy: an unusual skin condition associated with kidney disease. Semin Dial 2003; 16: 276-80.

16. Leiferman K. Cutaneous eosinophilic diseases. En: Freedberg I, Eisen A, Wolff K, Austen KF, Goldsmith L, Katz S, et al, editors. Fitzpatrick's dermatology in general medicine. $5^{\text {th }}$ ed. New York: McGraw-Hill; 1999; p. 1133-4.

17. Sidransky H. Eosinophilia myalgia syndrome: a recent syndrome serving as an alert to new diseases ahead. Mod Pathol 1994; 7: 806-10.

18. Greenburg as, Takagi H, Hill R, Hasan A, Murata H, FAlanga V. Delayed onset of skin fibrosis after the ingestion of eosinophilia myalgia syndromeassociated L-tryptophan. J Am Acad Dermatol 1996; 35: 264-6.

19. Quan Te, Cowper SE, Wu SP, Bockenstedt LK, Bucala R. Circulating fibrocytes: collagen-secreting cells of peripheral blood. Int J Biochem Cell Biol 2004; 36: 598-606.

20. Cowper SE. Nephrogenic systemic fibrosis: the nosological and conceptual evolution of Nephrogenic fibrosing dermopathy. Am J Kidney Dis 2005; 46: 763-65.

21. Hubbard V, Davenport A, Jarmulowicz M, Rustin M. Scleromyxoedema-like changes in four renal dialysis patients. Br J Dermatol 2003; 148: 563-68.

22. Fazeli A, Lio PA, Liu V. Nephrogenic fibrosing dermopathy: are ACE inhibitors the missing link? Arch Dermatol 2004; 140: 1401.
23. Baron PW, Cantos K, Hillebrand DJ, Hu KQ, Ojogho ON, Nehlsen-Cannarella S. Nephrogenic fibrosing dermopathy after liver transplantation successfully treated with plasmapheresis. Am J Dermatopathol 2003; 25: 204-09.

24. Cowper SE, Bucala R, Leboit PE. Nephrogenic fibrosing dermopathy/nephrogenic systemic fibrosis-setting the record straight. Semin Arthritis Rheum 2006; 35: 208-10.

25. Grobner T. Gadolinium - a specific trigger for the development of nephrogenic fibrosing dermopathy and nephrogenic systemic fibrosis? Nephrol Dial Transplant 2006; 21: 1104-8.

26. High AW, Ayers RA, Chandler J, Zito G, Cowper S. Gadolinium is detectable within the tissue of patients with nephrogenic systemic fibrosis. J Am Acad Dermatol 2007; 56: 21-26.

27. Swartz RD, Crofford LJ, Phan SH, Ike RW, Su LD. Nephrogenic Fibrosing Dermopathy: A novel cutaneous fibrosing disorder in patients with renal failure. Am J Med 2003; 114: 563-72.

28. Hauser C, Kaya G, Chizzolini C. Nephrogenic fibrosing dermopathy in a renal transplant recipient with tubulointerstitial nephritis and uveitis. Dermatology 2004; 209: 50-2.

29. Lauchli S, Zortea-Caflisch C, Nestle FO, Burg G, KempF W. Nephrogenic fibrosing dermopathy treated with extracorporeal photopheresis. Dermatology 2004; 208: 278-80.

30. Jan F, Segal JM, Dyer J, LeBoit P, Siegfried E, FrIEDEN IJ. Nephrogenic fibrosing dermopathy: two pediatric cases. J Pediatr 2003; 143: 678-81.

31. Chung HJ, Chung KY. Nephrogenic fibrosing dermopathy: response to high-dose intravenous immunoglobulin. Br J Dermatol 2004; 150: 59697.

32. Gilliet M, Cozzio A, Burg G, Nestle FO. Successful treatment of three cases of nephrogenic fibrosing dermopathy with extracorporeal photopheresis. Br J Dermatol 2005; 152: 531-6. 